\title{
REPROdUCTIVE HISTORY AND RETIREMENT: GENDER DifFERENCES AND V ARIATIONS Across WeLFARE STATES
}

Karsten Hank, Julie M. Korbmacher 


\title{
14 Reproductive History and Retirement: Gender Differences and Variations Across Welfare States
}

\author{
Karsten Hank and Julie M. Korbmacher
}

\subsection{Multiple perspectives on the fertility-employment nexus in later life}

The association between women's fertility and employment has received considerable attention in the social science literature, which documents significant variation in the observed correlations across time and between welfare states (Ahn \& Mira, 2002). Moreover, the employment-fertility-nexus has been shown to vary by gender: mothers might suffer from limited opportunities for paid employment under the same institutional regime that allows for positive income effects of fatherhood, for example.

Parenthood and its immediate consequences for labour market outcomes are also likely to exhibit sustained influences on employment patterns over the family life course, particularly for women (Brugiavini in chapter 13 of this volume). Even though children need not necessarily reduce one's total years in the labour force, they may create discontinuities in employment careers eventually affecting individuals' pension receipt. Thus "the retirement process is appropriately viewed as temporarily embedded in current incentive-disincentive structures that mediate retirement decision-making and in long-term family relations that constitute the joint role pathways of couples through work and family domains" (O'Rand et al., 1992: 82; italics in the original).

Only few studies have yet investigated the long-term relationship between individuals' reproductive history and retirement, though. The limited evidence collected so far tends to suggest that having children might actually delay women's exit from the work force (Hank, 2004). These and related findings have been interpreted as reflections of gendered role patterns developed across the family life course (Henretta et al., 1993; Pienta et al., 1994), but they are also likely to be affected by welfare state policies and institutions - which are not gender neutral either (Daly \& Rake, 2003).

Students of the interplay between childbearing and later life labour force exits should thus ideally take a cross-national and gendered perspective. With its broad set of retrospective life history information from respondents in 13 Continental European countries, SHARELIFE offers unique opportunities for researchers to conduct such kind of analysis, allowing us to add to the existing literature by providing initial answers to three important questions in particular: 
- Are there systematic gender differences in the association between reproductive history and retirement?

- Are longstanding differences in fertility, employment, pensions, and welfare regimes reflected in cross-national differences in the association between individuals' reproductive history and retirement? One might assume, for example, that institutional contexts fostering younger mothers' employment (e.g. Sweden) and such prohibiting women's labour force participation after childbirth (e.g. Italy) also exhibit such patterns if older women's employment is considered.

- Are there cohort differences in the association between reproductive history and retirement?

\subsection{Reproductive and employment histories in SHARELIFE}

In our study, we track the annual employment histories of 16,454 ever married men and women aged 50 or older who reported at least one episode of gainful employment. These individuals contribute a total of 156,033 person-years of observation to the analysis. Running separate regressions for men and women, we estimate discrete-time logit models to analyse the individual's entry into retirement.

For our dependent variable 'retirement' there is no exclusive definition. It is operationalised here as an exit from the labour force after age 50 which is not followed by a re-entry into paid employment in any subsequent time period (note that observations are censored at age 80 or at the time of the SHARELIFE interview); Blanchet et al. (2005) for an overview of different pathways to retirement in SHARE countries.

Our main explanatory variables are three indicators of individuals' fertility: first, the number of children ever born (with an average of 2.1 in our sample); second, a dummy variable indicating whether the individual ever had a child (note that $8-9 \%$ of the respondents in our sample remained childless); and third, binary indicators of an early (late, respectively) first birth, where the threshold was set at age 24 for women and age 27 for men (i.e. the median of the distribution for the pooled sample). Such detailed new information on men's and women's reproductive history substantially increases SHARE's research potential in this domain (see Martínez-Granado \& Mira, 2005, for an analysis of fertility using data from SHARE's Wave 1).

Further control variables include individuals' age, level of education, work experience (years in the labour force and number of jobs till the age of 50), marital status as well as indicators of self-employment and homeownership (Hank, 2004). To account for possible cohort differences in the relationship between fertility and retirement, we distinguish between respondents born up until 1940 (who account for roughly one third of our sample) and those born later. 
For our regionalized analyses, we use Esping-Andersen's (1990) initial welfare state typology to group the countries represented in SHARELIFE into four clusters which we label as Social-democratic (DK, NL, SE), Conservative (AT, BE, CH, DE, FR), Mediterranean (ES, GR, IT), and Post-communist (CZ, PL).

\subsection{Parenthood, number of children, timing of fertility - what determines older individuals' exit from the labour market?}

Before turning to the discussion of the main explanatory variables, which also considers cohort and welfare regime interactions (see Table 14.2), we first describe the results for all other control variables in an initial model for the pooled SHARELIFE sample (Table 14.1). Most of these findings are consistent with previous research (Hank, 2004) and very similar for men and women.

To begin with, the odds of entering retirement increases significantly with individuals' age, sky-rocketing after age 59 and yet again at ages 64 and over. Moreover, members of the post-1940 cohorts retire later than those born before World War II. With regard to the role of a woman's marital status, there is clear indication that married women enter retirement earlier than their unmarried counterparts, whereas the respective coefficient remains insignificant for men. This finding might be interpreted as evidence supporting the 'joint retirement hypothesis' (Henretta et al., 1993), which states that (on average older) husbands and (on average younger) wives tend to retire at the same time, resulting in earlier retirement among married women.

Table 14.1: Discrete-time logistic regression results for older women's and men's exit from the labour force, odds ratios (standard errors) - pooled initial model

\begin{tabular}{lrr}
\hline Demographics & Women & Men \\
\hline & & \\
Age 50-53a & 1.00 & 1.00 \\
Age 54-57 & $2.92^{* * *}$ & $2.52^{* * *}$ \\
& $(0.136)$ & $(0.124)$ \\
Age 58-59 & $4.20^{* * *}$ & $4.71^{* * *}$ \\
& $(0.252)$ & $(0.259)$ \\
Age 60-61 & $14.55^{* * *}$ & $15.83^{* * *}$ \\
& $(0.819)$ & $(0.806)$ \\
Age 62-63 & $13.06^{* * *}$ & $13.47^{* * *}$ \\
& $(0.916)$ & $(0.804)$ \\
Age 64+ & $19.12^{* * *}$ & $29.89^{* * *}$ \\
\hline
\end{tabular}




\begin{tabular}{|c|c|c|}
\hline & (1.310) & $(1.704)$ \\
\hline \multirow[t]{2}{*}{ Born 1940 or later } & $0.82 * * *$ & $0.93 * *$ \\
\hline & $(0.028)$ & (0.028) \\
\hline \multirow[t]{2}{*}{ Married } & $1.12 * * *$ & 1.08 \\
\hline & $(0.046)$ & $(0.057)$ \\
\hline \multicolumn{3}{|l|}{ Education \& work } \\
\hline Low educationa & 1.00 & 1.00 \\
\hline \multirow[t]{2}{*}{ Medium education } & $0.91^{* *}$ & 1.05 \\
\hline & $(0.035)$ & $(0.039)$ \\
\hline \multirow[t]{2}{*}{ High education } & $0.70 * * *$ & 0.96 \\
\hline & $(0.034)$ & $(0.044)$ \\
\hline \multirow[t]{2}{*}{ Years in labour force at age 50} & $1.02 * * *$ & $1.07^{* * *}$ \\
\hline & $(0.002)$ & $(0.004)$ \\
\hline \multirow[t]{2}{*}{ Number of jobs at age 50} & 1.00 & 1.00 \\
\hline & $(0.010)$ & $(0.009)$ \\
\hline \multirow[t]{2}{*}{ Last job self-employed } & $0.42 * * *$ & $0.37^{* * *}$ \\
\hline & $(0.022)$ & $(0.015)$ \\
\hline \multirow[t]{2}{*}{ Homeowner } & 0.95 & 1.03 \\
\hline & $(0.034)$ & $(0.034)$ \\
\hline \multicolumn{3}{|c|}{ Reproductive history (also see Table 2) } \\
\hline \multirow[t]{2}{*}{ Number of children } & 1.00 & $0.98^{*}$ \\
\hline & $(0.013)$ & $(0.011)$ \\
\hline \multirow[t]{2}{*}{ Constant } & $0.02 * * *$ & $0.00 * * *$ \\
\hline & $(0.002)$ & $(0.000)$ \\
\hline Pseudo-R2 & .13 & .16 \\
\hline Observations & 64,528 & 91,446 \\
\hline
\end{tabular}

Women with a high educational degree tend to stay in the labour force longer than their low qualified counterparts. The number of years in employment till the age of 50 significantly influences the individual's decision to retire. A longer employment record results in earlier eligibility for old-age pensions and tends to speed up the transition to retirement. Moreover, those who were self-employed in their last reported job exhibit markedly lower risks of leaving the labour force than all others. Career interruptions (indicated by the number of employment spells) as well as homeownership, however, do not bear any association with the timing of retirement.

Table 14.2 summarises the results for different indicators of men's and women's reproductive history. In the initial model (also exhibited in Table 14.1) we operationalised individuals' reproductive history by their number of children. The risk of entering retirement among women born 1940 or later tends to slightly 
increase with a growing number of children (Table 14.2a). This finding seems to be driven mainly by women living under social-democratic and post-communist regimes. The reverse association is observed among men (Table 14.2e). An alternative specification, based on an indicator of whether the respondent ever had a child, also suggests that, independent of family size, in cohorts born post-1940 fatherhood (at least in social-democratic countries) bears a negative correlation with retirement (Table 14.2f), whereas motherhood contributes to an earlier exit from the labour force (Table 14.2b). Moreover, mothers who experienced an early first birth (particularly if they live in a conservative country) retire earlier than their childless counterparts (Table 14.2c), a result which - again - appears to be driven mainly by 'younger' cohorts of women. Conversely, 'late' fathers living under a social-democratic regime are shown to retire later than childless men (Table 14.2h).

Table 14.2: Discrete-time logistic regression results for older women's and men's exit from the labour force, odds ratios (standard errors) - Interaction of reproductive history indicators with cohort and welfare state regime

\begin{tabular}{|c|c|c|c|c|c|c|}
\hline \multirow{3}{*}{$\begin{array}{l}\text { Women } \\
\text { Welfare } \\
\text { regimes }\end{array}$} & \multicolumn{3}{|c|}{ (a) Number of children } & \multicolumn{3}{|c|}{ (b) Ever had a child } \\
\hline & \multirow[t]{2}{*}{ All } & \multicolumn{2}{|c|}{ Cohort } & \multirow[t]{2}{*}{ All } & \multicolumn{2}{|c|}{ Cohort } \\
\hline & & $<1940$ & $>1940$ & & $<1940$ & $>1940$ \\
\hline $\begin{array}{l}\text { Social- } \\
\text { democratic }\end{array}$ & $\begin{array}{r}1.07^{* *} \\
(0.03)\end{array}$ & $\begin{array}{r}1.06 \\
(0.04)\end{array}$ & $\begin{array}{r}1.11^{* *} \\
(0.05)\end{array}$ & $\begin{array}{r}1.09 \\
(0.13)\end{array}$ & $\begin{array}{r}1.05 \\
(0.18)\end{array}$ & $\begin{array}{r}1.18 \\
(0.20)\end{array}$ \\
\hline Conservative & $\begin{array}{r}1.00 \\
(0.02)\end{array}$ & $\begin{array}{r}0.97 \\
(0.03)\end{array}$ & $\begin{array}{r}1.02 \\
(0.03)\end{array}$ & $\begin{array}{r}1.06 \\
(0.10)\end{array}$ & $\begin{array}{r}0.85 \\
(0.12)\end{array}$ & $\begin{array}{r}1.19 \\
(0.16)\end{array}$ \\
\hline Mediterranean & $\begin{array}{r}0.96 \\
(0.03)\end{array}$ & $\begin{array}{r}0.96 \\
(0.04)\end{array}$ & $\begin{array}{r}0.97 \\
(0.05)\end{array}$ & $\begin{array}{r}1.17 \\
(0.16)\end{array}$ & $\begin{array}{r}1.06 \\
(0.20)\end{array}$ & $\begin{array}{r}1.29 \\
(0.26)\end{array}$ \\
\hline $\begin{array}{l}\text { Post- } \\
\text { communist }\end{array}$ & $\begin{array}{r}1.04 \\
(0.03)\end{array}$ & $\begin{array}{r}1.00 \\
(0.05)\end{array}$ & $\begin{array}{r}1.11^{* *} \\
(0.05)\end{array}$ & $\begin{array}{r}1.04 \\
(0.17)\end{array}$ & $\begin{array}{r}0.85 \\
(0.22)\end{array}$ & $\begin{array}{r}1.18 \\
(0.26)\end{array}$ \\
\hline All & $\begin{array}{r}1.00 \\
(0.01)\end{array}$ & $\begin{array}{r}0.98 \\
(0.02)\end{array}$ & $\begin{array}{c}1.04^{*} \\
(0.02)\end{array}$ & $\begin{array}{r}1.09 \\
(0.07)\end{array}$ & $\begin{array}{r}0.95 \\
(0.08)\end{array}$ & $\begin{array}{c}1.21^{* *} \\
(0.10)\end{array}$ \\
\hline
\end{tabular}

\begin{tabular}{|c|c|c|c|c|c|c|}
\hline \multirow{3}{*}{$\begin{array}{l}\text { Women } \\
\text { Welfare } \\
\text { regimes }\end{array}$} & \multicolumn{3}{|c|}{ (c) Early first birth } & \multicolumn{3}{|c|}{ (d) Late first birth } \\
\hline & \multirow[t]{2}{*}{ All } & \multicolumn{2}{|c|}{ Cohort } & \multirow[t]{2}{*}{ All } & \multicolumn{2}{|c|}{ Cohort } \\
\hline & & $<1940$ & $>1940$ & & $<1940$ & $>1940$ \\
\hline \multirow{2}{*}{$\begin{array}{l}\text { Social- } \\
\text { democratic }\end{array}$} & 1.22 & 1.25 & 1.21 & 1.07 & 1.11 & 1.05 \\
\hline & $(0.15)$ & $(0.22)$ & $(0.21)$ & $(0.13)$ & $(0.19)$ & $(0.19)$ \\
\hline \multirow[t]{2}{*}{ Conservative } & 1.16 & 0.92 & $1.33^{* *}$ & 0.98 & 0.88 & 1.00 \\
\hline & $(0.11)$ & $(0.13)$ & $(0.18)$ & $(0.10)$ & $(0.13)$ & $(0.14)$ \\
\hline \multirow[t]{2}{*}{ Mediterranean } & 1.12 & 0.99 & 1.27 & 1.20 & 1.12 & 1.27 \\
\hline & $(0.16)$ & $(0.20)$ & $(0.27)$ & $(0.17)$ & $(0.22)$ & $(0.26)$ \\
\hline
\end{tabular}




\begin{tabular}{|c|c|c|c|c|c|c|}
\hline $\begin{array}{l}\text { Post- } \\
\text { communist }\end{array}$ & $\begin{array}{r}1.17 \\
(0.18)\end{array}$ & $\begin{array}{r}1.03 \\
(0.25)\end{array}$ & $\begin{array}{r}1.27 \\
(0.27)\end{array}$ & $\begin{array}{r}1.00 \\
(0.17)\end{array}$ & $\begin{array}{r}1.07 \\
(0.28)\end{array}$ & $\begin{array}{r}0.99 \\
(0.22)\end{array}$ \\
\hline All & $\begin{array}{r}1.17^{* * * *} \\
(0.07)\end{array}$ & $\begin{array}{r}1.04 \\
(0.09)\end{array}$ & $\begin{array}{r}1.29 * * * \\
(0.11)\end{array}$ & $\begin{array}{r}1.06 \\
(0.07)\end{array}$ & $\begin{array}{r}1.03 \\
(0.09)\end{array}$ & $\begin{array}{r}1.08 \\
(0.09) \\
\end{array}$ \\
\hline Men & \multicolumn{3}{|c|}{ (e) Number of children } & \multicolumn{3}{|c|}{ (f) Ever had a child } \\
\hline Welfare & All & \multicolumn{2}{|c|}{ Cohort } & All & \multicolumn{2}{|c|}{ Cohort } \\
\hline regimes & & $<1940$ & $>1940$ & & $<1940$ & $>1940$ \\
\hline $\begin{array}{l}\text { Social- } \\
\text { democratic }\end{array}$ & $\begin{array}{r}0.97 \\
(0.02)\end{array}$ & $\begin{array}{r}0.98 \\
(0.03)\end{array}$ & $\begin{array}{r}0.94 \\
(0.04)\end{array}$ & $\begin{array}{r}0.86 \\
(0.09)\end{array}$ & $\begin{array}{r}0.97 \\
(0.15)\end{array}$ & $\begin{array}{r}0.73^{* *} \\
(0.11)\end{array}$ \\
\hline Conservative & $\begin{array}{r}0.98 \\
(0.02)\end{array}$ & $\begin{array}{r}0.99 \\
(0.02)\end{array}$ & $\begin{array}{r}0.96 \\
(0.03)\end{array}$ & $\begin{array}{r}1.06 \\
(0.09)\end{array}$ & $\begin{array}{r}1.11 \\
(0.13)\end{array}$ & $\begin{array}{r}0.98 \\
(0.13)\end{array}$ \\
\hline Mediterranean & $\begin{array}{r}0.97 \\
(0.02)\end{array}$ & $\begin{array}{r}0.97 \\
(0.03)\end{array}$ & $\begin{array}{r}0.97 \\
(0.04)\end{array}$ & $\begin{array}{r}0.96 \\
(0.10)\end{array}$ & $\begin{array}{r}0.88 \\
(0.13)\end{array}$ & $\begin{array}{r}1.01 \\
(0.15)\end{array}$ \\
\hline $\begin{array}{l}\text { Post- } \\
\text { communist }\end{array}$ & $\begin{array}{r}1.01 \\
(0.03)\end{array}$ & $\begin{array}{r}1.07 \\
(0.05)\end{array}$ & $\begin{array}{r}0.97 \\
(0.05)\end{array}$ & $\begin{array}{r}1.22 \\
(0.23)\end{array}$ & $\begin{array}{r}1.23 \\
(0.32)\end{array}$ & $\begin{array}{r}1.24 \\
(0.35)\end{array}$ \\
\hline All & $\begin{array}{l}0.98^{*} \\
(0.01)\end{array}$ & $\begin{array}{r}0.99 \\
(0.01)\end{array}$ & $\begin{array}{l}0.97^{*} \\
(0.02)\end{array}$ & $\begin{array}{r}1.00 \\
(0.05)\end{array}$ & $\begin{array}{r}1.04 \\
(0.08)\end{array}$ & $\begin{array}{r}0.95 \\
(0.07) \\
\end{array}$ \\
\hline Men & \multicolumn{3}{|c|}{ (c) Early first birth } & \multicolumn{3}{|c|}{ (d) Late first birth } \\
\hline Welfare & All & \multicolumn{2}{|c|}{ Cohort } & All & \multicolumn{2}{|c|}{ Cohort } \\
\hline regimes & & $<1940$ & $>1940$ & & $<1940$ & $>1940$ \\
\hline $\begin{array}{l}\text { Social- } \\
\text { democratic }\end{array}$ & $\begin{array}{r}0.93 \\
(0.10)\end{array}$ & $\begin{array}{r}1.02 \\
(0.15)\end{array}$ & $\begin{array}{r}0.81 \\
(0.13)\end{array}$ & $\begin{array}{r}0.78^{* *} \\
(0.09)\end{array}$ & $\begin{array}{r}0.94 \\
(0.14)\end{array}$ & $\begin{array}{r}0.62^{* * *} \\
(0.10)\end{array}$ \\
\hline Conservative & $\begin{array}{r}1.12 \\
(0.10)\end{array}$ & $\begin{array}{r}1.19 \\
(0.14)\end{array}$ & $\begin{array}{r}1.00 \\
(0.13)\end{array}$ & $\begin{array}{r}1.01 \\
(0.09)\end{array}$ & $\begin{array}{r}1.04 \\
(0.13)\end{array}$ & $\begin{array}{r}0.94 \\
(0.13)\end{array}$ \\
\hline Mediterranean & $\begin{array}{r}1.05 \\
(0.11)\end{array}$ & $\begin{array}{r}0.96 \\
(0.15)\end{array}$ & $\begin{array}{r}1.09 \\
(0.17)\end{array}$ & $\begin{array}{r}0.92 \\
(0.10)\end{array}$ & $\begin{array}{r}0.89 \\
(0.13)\end{array}$ & $\begin{array}{r}0.95 \\
(0.14)\end{array}$ \\
\hline $\begin{array}{l}\text { Post- } \\
\text { communist }\end{array}$ & $\begin{array}{r}1.24 \\
(0.22)\end{array}$ & $\begin{array}{r}1.28 \\
(0.32)\end{array}$ & $\begin{array}{r}1.23 \\
(0.33)\end{array}$ & $\begin{array}{r}1.25 \\
(0.23)\end{array}$ & $\begin{array}{r}1.26 \\
(0.33)\end{array}$ & $\begin{array}{r}1.22 \\
(0.34)\end{array}$ \\
\hline All & $\begin{array}{r}1.05 \\
(0.06)\end{array}$ & $\begin{array}{r}1.10 \\
(0.08)\end{array}$ & $\begin{array}{r}0.99 \\
(0.08)\end{array}$ & $\begin{array}{r}0.94 \\
(0.05)\end{array}$ & $\begin{array}{r}1.00 \\
(0.08)\end{array}$ & $\begin{array}{r}0.88 \\
(0.07)\end{array}$ \\
\hline
\end{tabular}

Note: Social-democratic: DK, NL, SE; Conservative: $\mathrm{AT}, \mathrm{BE}, \mathrm{CH}, \mathrm{DE}, \mathrm{FR}$; Mediterranean: ES, GR, IT; Post-communist: CZ, PL. All control variables used in the initial model (cf. Table 2) are also included here. Significance: ${ }^{* * *}=1 \%$; ${ }^{* *}=5 \%{ }^{*}=10 \%$. 


\subsection{Summary}

The main findings of our initial assessment of the association between men's and women's reproductive history and retirement in Continental Europe can be summarised as follows:

- Different from previous research for Germany (Hank, 2004), our findings based on SHARELIFE suggest that mothers (just as married women) are more likely than childless women to exit the labour force early, whereas fathers tend to retire later than other men. These findings might, in general, be interpreted as a reflection of a weaker labour force attachment among mothers and a greater responsibility of fathers as male breadwinners lasting well into individuals' late career phase.

- However, the association between childbearing and earlier retirement appears to be particularly strong among women living under a socialdemocratic or post-communist welfare state regime, that is, in countries exhibiting relatively high levels of female labour force participation. This might indicate that mothers in conservative and Mediterranean countries who re-entered the labour market after childbirth may be a selective group characterised by an above average attachment to employment. To investigate further the role of specific welfare state interventions (particularly such supporting mothers' employment or granting them special pension entitlements) is beyond the scope of this paper and therefore remains an important task for future research.

- There is some indication for a closer relationship between individuals' reproductive history and retirement decisions among 'younger' cohorts born 1940 or later. This is likely to indicate changes in the work-family nexus across time, which clearly deserves further attention.

\section{References}

Ahn, N. \& Mira, P. (2002): A note on the changing relationship between fertility and female employment rates in developed countries. Journal of Population Economics, Vol. 15, pp. 667682.

Blanchet, D., Brugiavini, A., \& Rainato, R. (2005): Pathways to retirement, in A. Börsch-Supan et al. (eds.), Health, Ageing and Retirement in Europe - First results from SHARE, Mannheim: MEA, pp. 246-252.

Daly, M. \& Rake, K. (2003): Gender and the welfare state, Cambridge: Polity Press.

Esping-Andersen, G. (1990): The three worlds of welfare capitalism, Princeton: Princeton University Press.

Hank, K. (2004): Effects of Early Life Family Events on Women's Late Life Labour Market Behaviour: An Analysis of the Relationship between Childbearing and Retirement in Western Germany. European Sociological Review, Vol. 20, pp. 189-198. 
Henretta, J.C., O'Rand, A. \& Chan, C.G. (1993): Joint role investments and synchronization of retirement: A sequential approach to couple's retirement timing. Social Forces, Vol. 71, pp. 981-1000.

Martínez-Granado, M. \& Mira, P. (2005): The number of living children, in A. Börsch-Supan et al. (eds.), Health, Ageing and Retirement in Europe - First results from SHARE, Mannheim: MEA, pp. 48-52.

O'Rand, A.M., Henretta, J.C., \& Krecker, M.L. (1992): Family Pathways to Retirement, in M. Szinovacz et al. (eds.), Families and Retirement: Conceptual and Methodological Issues, Beverly Hills: Sage, pp. 81-98.

Pienta, A.M., Burr, J.A., \& Mutchler, J.E. (1994): Women's labor force participation in later life: The effects of early work and family experiences. Journal of Gerontology: Social Sciences, Vol. 49, pp. 231-239. 